EPJ Web of Conferences 86, 00058 (2015)

DOI: $10.1051 /$ epjconf/ 20158600058

(C) Owned by the authors, published by EDP Sciences, 2015

\title{
Role of the Skyrme tensor force in heavy-ion fusion
}

\author{
P. D. Stevenson ${ }^{1}$, E. B. Suckling ${ }^{1}$, S. Fracasso ${ }^{1}$, E. D. Simmons ${ }^{1}$ and A. S. Umar ${ }^{2}$ \\ ${ }^{1}$ Department of Physics, University of Surrey, Guildford, Surrey, GU2 7XH, United Kingdom \\ ${ }^{2}$ Department of Physics and Astronomy, Vanderbilt University, Nashville, Tennessee 37235, USA
}

\begin{abstract}
We make use of the Skyrme effective nuclear interaction within the time-dependent Hartree-Fock framework to assess the effect of inclusion of the tensor terms of the Skyrme interaction on the fusion window of the ${ }^{16} \mathrm{O}-{ }^{16} \mathrm{O}$ reaction. We find that the lower fusion threshold, around the barrier, is quite insensitive to these details of the force, but the higher threshold, above which the nuclei pass through each other, changes by several $\mathrm{MeV}$ between different tensor parametrisations. The results suggest that eventually fusion properties may become part of the evaluation or fitting process for effective nuclear interactions.
\end{abstract}

\section{Introduction}

The Skyrme interaction was introduced in the 1950s [1] and has become the most widespread effective interaction used in mean-field calculations in nuclei. Its utility lies in part in the fact that it is designed as a kind of series expansion around a zero-range interaction; this gives a delta function in each term, though with spatial derivatives to explore the finite range part of the nuclear interaction, just as a Taylor expansion of a function is able to converge on true values of that function away from the point of expansion through the use of derivatives. The delta functions afford great calculational simplifications, and also allow a straightforward transformation between the effective interaction picture and an energy density functional.

As originally formulated, the Skyrme effective interaction featured tensor terms, reflecting their known importance in the underlying nucleon-nucleon interaction. The tensor terms had subsequently been widely neglected (as the fits made at the level of Hartree-Fock calculations were not very sensitive to the tensor terms). More recently, a renewed interest in the role of the tensor terms has arisen [2-8], as the ability to perform large-scale calculations of nuclear properties, in which the tensor term may show an effect, have become the norm.

Various parametrisations of the Skyrme interaction in which tensor terms are active have been produced. We select a small sample for this work to show the variation of results. We use a standard time-dependent Hartree-Fock (TDHF) implementation, based on the Sky3D code [9], with all the extra time-even and time-odd terms, including those from the tensor force (see [7] and references therein). The code also works as a standard static Hartree-Fock code to initialise the time-dependent run with nuclear ground states obtained via a damped relaxation method [10]. We note here that the tensor force does not merely amend the coefficients at the level of the energy density functional, but adds further couplings between densities [8]. Further work awaits a longer subsequent publication, or can be found in the $\mathrm{PhD}$ thesis of one of us [12]. For more details of TDHF and its extensions, including its use in fusion reactions, the recent review by Simenel is a useful source [13].

\section{The Skyrme tensor force}

We use the tensor terms as introduced by Skyrme [1] (though written in the notation of [2]):

$$
\begin{aligned}
V_{t}\left(\boldsymbol{r}_{1}, \boldsymbol{r}_{2}\right)= & \frac{t_{e}}{2}\left(\left[3\left(\hat{\boldsymbol{\sigma}}_{1} \cdot \boldsymbol{k}^{\prime}\right)\left(\hat{\boldsymbol{\sigma}}_{2} \cdot \boldsymbol{k}^{\prime}\right)-\right.\right. \\
& \left.\left(\hat{\boldsymbol{\sigma}}_{1} \cdot \hat{\boldsymbol{\sigma}}_{2}\right) \boldsymbol{k}^{\prime 2}\right] \delta\left(\boldsymbol{r}_{1}-\boldsymbol{r}_{2}\right) \\
+ & \delta\left(\boldsymbol{r}_{1}-\boldsymbol{r}_{2}\right)\left[3\left(\hat{\boldsymbol{\sigma}}_{1} \cdot \boldsymbol{k}\right)\left(\hat{\boldsymbol{\sigma}}_{2} \cdot \boldsymbol{k}\right)-\right. \\
& \left.\left.\left(\hat{\boldsymbol{\sigma}}_{1} \cdot \hat{\boldsymbol{\sigma}}_{2}\right) \boldsymbol{k}^{2}\right]\right) \\
+ & t_{o}\left[3\left(\hat{\boldsymbol{\sigma}}_{1} \cdot \boldsymbol{k}^{\prime}\right) \delta\left(\boldsymbol{r}_{1}-\boldsymbol{r}_{2}\right)\left(\hat{\boldsymbol{\sigma}}_{2} \cdot \boldsymbol{k}\right)\right. \\
& \left.-\left(\hat{\boldsymbol{\sigma}}_{1} \cdot \hat{\boldsymbol{\sigma}}_{2}\right) \boldsymbol{k}^{\prime} \delta\left(\boldsymbol{r}_{1}-\boldsymbol{r}_{2}\right) \boldsymbol{k}\right] .
\end{aligned}
$$

This term contains two parameters to be fitted to data; $t_{e}$ and $t_{o}$. Here the subscript $e_{e}$ means even and ${ }_{o}$ means $o d d$, since the associated terms are respectively even and odd under the exchange of spatial coordinates.

\section{Fusion Windows}

Calculations were performed using our modified Sky3D code for the upper fusion thresholds at zero impact parameter - i.e. the highest energy at which two ${ }^{16} \mathrm{O}$ nuclei fuse during head-on collisions, above which the nuclei pass through and undergo deep-inelastic excitation. We choose the forces SV-bas [14], as a sample non-tensor force (and that which comes as the default with the Sky3D sample input files), SLy5 [15], as a force whose time-odd terms have 
been explored in previous TDHF calculations [16], and which has independently seen the perturbative addition of tensor terms, fitted to single-particle energy systematics [11] while leaving the rest of the force unchanged. The forces T22, T24 and T26 [2] had the tensor terms included in the initial fit, and are part of a series of forces with systematic variation of the tensor properties. Here, the variation of the second numeral in the force name indicates that the $\mathrm{p}-\mathrm{n}$ part of the tensor term in the effective interaction is systematically varying.

The choice of a ${ }^{16} \mathrm{O}+{ }^{16} \mathrm{O}$ reaction comes from its long history of use as a kind of standard system against which new techniques of forces are benchmarked[17]. Selected examples of such recent studies of ${ }^{16} \mathrm{O}+{ }^{16} \mathrm{O}$ fusion reactions include the analysis of equilibriation [18], a detailed microscopic study around the barrier [19], and a study of the effect of the time-odd couplings in the spin-orbit force on dissipation as a function of energy [20], also including tensor-force contributions to the spin-orbit part of the energy density functional [21]. Other recent applications of TDHF include a study of dynamic effects on potential barriers for heavier systems [22], and the implementation of a continuum-TDHF theory [23].

\begin{tabular}{cc}
\hline Force & Threshold (MeV) \\
\hline \hline SV-bas & 75 \\
SLy5 (full) & 68 \\
SLy5 (tensor) & 65 \\
$T 22$ & 63 \\
$T 24$ & 71 \\
$T 26$ & 84 \\
\hline
\end{tabular}

Table 1. Fusion upper threshold energies for the ${ }^{16} \mathrm{O}+{ }^{16} \mathrm{O}$ collision using various parametrisations of the Skyrme interaction. For references to interactions, see text. The energies are calculated to the nearest $1 \mathrm{MeV}$.

The results for the maximum fusing energy are given for this selection of forces in table 1. We label SLy5 (full) as the SLy5 force as originally conceived, with no tensor force, but with time-odd terms active where they arise from the original Skyrme parameters, with the exception of the $(\vec{\nabla} \cdot \vec{s})^{2}$ and $\vec{s} \cdot \nabla^{2} \vec{s}$ which may result in spin instabilities. It is seen that, at least in the case of SLy5 that adding the tensor terms to the force decreases the upper fusion threshold. This means that the tensor terms in the effective interaction serve to decrease transfer of relative kinetic energy of the fragments into their internal energy during the reaction. If this can be explained in terms of the sign of the tensor terms, which were determined solely by the requirement to improve the single particle energies [11], an important constraint for the tensor force would be found. Despite SV-bas and SLy5 both being fitted to group state properties of finite nuclei and to nuclear matter properties, a rather large difference in upper threshold energy is evident. The series of TIJ forces then shows a very wide range of energy differences for the upper threshold, despite all producing similar and reasonable ground state properties. We should point out that the lower (barrier) thresholds for fusion are quite insensitive to the Skyrme parametrisation, at least to the level of around $1 \mathrm{MeV}$ difference between forces, with the main effect being due to the Coulomb force.

\section{Conclusions}

We have performed time-dependent Hartree-Fock calculations of fusion reactions between two ${ }^{16} \mathrm{O}$ nuclei, using a range of different parametrisations of the effective Skyrme interactions. Each of the interactions produces rather similar ground states in which neither time-odd nor tensor parts of the effective interaction are active, but the range of results in the upper fusion threshold is large. This highlights the as-yet unconstrained nature of the time-odd parts of the effective nuclear interaction. While systematic use of fusion calculations in fits of time-odd parts of Skyrme forces remains computationally prohibitive, the variation found here suggests that fusion dynamics may form part of the physics input to future constraints on those parts of effective interactions which are not probed by ground-state fits. This can be added to the increasing body of work indicating that the time-odd contributions to the nuclear meanfield are in need of constraining to observables [24-26].

\section{Acknowledgements}

This work was funded in part by the UK Science and Technology Facility Council under grant nos ST/J000051/1, ST/F500613/1, ST/L005816/1 and PP/F000596/1, running time granted by STFC on the DiRAC computer cluster and by the US Department of Energy under grant DE-FG0296ER40975 with Vanderbilt University

\section{References}

[1] T. H. R. Skyrme, Nucl. Phys. 9, 615 (1959)

[2] T. Lesinski, M. Bender, K. Bennaceur, T. Duguet, and J. Meyer, Phys. Rev. C 76, 014312 (2007)

[3] M. Bender, K. Bennaceur, T. Duguet, P.-H. Heenen, T. Lesinski, and J. Meyer, Phys. Rev. C 80, 064302 (2009)

[4] E. B. Suckling and P. D. Stevenson, EPL, 90, 12001 (2010)

[5] Xian-Rong Zhou and H. Sagawa, J. Phys. G: Nucl. Part. Phys. 39, 085104 (2012)

[6] A. Pastore, D. Davesne, Y. Lallouet, M. Martini, K. Bennaceur, and J. Meyer, Phys. Rev. C 85, 054317 (2012)

[7] S. Fracasso, E. B. Suckling, and P. D. Stevenson, Phys. Rev. C 86, 044303 (2012)

[8] P. D. Stevenson, S. Fracasso, and E. B. Suckling, J. Phys. Conf. Ser. 381, 012105 (2012)

[9] J. A. Maruhn, P.-G. Reinhard, P. D. Stevenson, and A. S. Umar, Comput. Phys. Commun. 85, 2195 (2014)

[10] C. Bottcher, M. R. Strayer, A. S. Umar, and P.-G. Reinhard, Phys. Rev. A 40, 4182 (1989)

[11] G. Colò, H. Sagawa, S. Fracasso, and P. F. Bortignon, Phys. Lett. B 646, 227 (2007); 668, 457(E) (2008) 
[12] E. B. Suckling, PhD thesis, University of Surrey (2011)

[13] C. Simenel, Eur. Phys. Jour. A 48, 152 (2012)

[14] P. Klüpfel, P.-G. Reinhard, T. J. Bürvenich, and J. A. Maruhn, Phys. Rev. C 79, 034310 (2009)

[15] E. Chabanat, P. Bonche, P. Haensel, J. Meyer, and R. Schaeffer, Nucl. Phys. A 635, 231 (1998)

[16] A. S. Umar and V. E. Oberacker, Phys. Rev. C 73, 054607 (2006)

[17] A. S. Umar, M. R. Strayer, and P.-G. Reinhard, Phys. Rev. Lett. 56, 2793 (1986)

[18] N. Loebl, J. A. Maruhn, and P.-G. Reinhard, Phys. Rev. C 84, 034608 (2011)

[19] C. Simenel, R. Keser, A. S. Umar, and V. E. Oberacker, Phys. Rev. C 88, 024617 (2013)
[20] G.-F. Dai, L. Guo, E.-G. Zhao, and S.-G. Zhou, Phys. Rev. C 90, 044609 (2014)

[21] G.-F. Dai, L. Guo, E.-G Zhao, and S.-G Zhou, Sci. China-Phys. Mech. Astron. 57, 1618 (2014)

[22] A. S. Umar, C. Simenel, and V. E. Oberacker, Phys. Rev. C 89, 034611 (2014)

[23] C. I. Pardi, P. D. Stevenson and K. Xu, Phys. Rev. E 89, 033312 (2014)

[24] L. M. Robledo, R. N. Bernard, and G. F. Bertsch, Phys. Rev. C 89, 021303(R) (2014)

[25] A. Pastore, D. Davasne, K. Bennaceur, J. Meyer, and V. Hellemans, Phys. Scr. T154, 014014 (2013)

[26] V. Hellemans, A. Pastore, T. Duguet, K. Bennaceur, D. Davesne, J. Meyer, M. Bender, and P.-H. Heenen, Phys. Rev. C 88, 064323 (2013) 